\title{
MULTIMODAL CONNECTOME BIOMARKERS OF COGNITIVE AND AFFECTIVE DYSFUNCTION IN THE COMMON EPILEPSIES
}

\author{
Raul Rodriguez-Cruces ${ }^{1 *}$, Jessica Royer ${ }^{1 *}$, Sara Larivière ${ }^{1}$, Dani S. Bassett ${ }^{2,3,4,5,6,7}$, \\ Lorenzo Caciagli ${ }^{2,8^{*}}$, Boris C Bernhardt ${ }^{1 *}$ \\ ${ }^{I}$ McConnell Brain Imaging Centre, Montreal Neurological Institute and Hospital, McGill University, Montreal, \\ Quebec, Canada; \\ ${ }^{2}$ Department of Bioengineering, University of Pennsylvania, Philadelphia, Pennsylvania 19104, USA \\ ${ }^{3}$ Department of Physics and Astronomy, University of Pennsylvania, Philadelphia, Pennsylvania 19104 USA \\ ${ }^{4}$ Department of Electrical and Systems Engineering, University of Pennsylvania, Philadelphia, Pennsylvania 19104 \\ USA \\ ${ }^{5}$ Department of Neurology, University of Pennsylvania, Philadelphia, Pennsylvania 19104 USA \\ ${ }^{6}$ Department of Psychiatry, University of Pennsylvania, Philadelphia, Pennsylvania 19104 USA \\ ${ }^{7}$ Santa Fe Institute, Santa Fe, New Mexico 87501 USA \\ ${ }^{8}$ Department of Clinical and Experimental Epilepsy, UCL Queen Square Institute of Neurology, London WC1N \\ $3 B G$, United Kingdom
}




\begin{abstract}
Epilepsy is one of the most common chronic neurological conditions, traditionally defined as a disorder of recurrent seizures. Cognitive and affective dysfunctions are increasingly recognized as core disease dimensions and can affect patient wellbeing sometimes more than the seizures themselves. Connectomebased approaches hold immense promise for revealing mechanisms that contribute to dysfunction, and to identify biomarkers. Our review discusses emerging multimodal neuroimaging and connectomics studies that highlight network substrates of cognitive/affective dysfunction in the common epilepsies. We first overview work in drug-resistant epilepsy syndromes i.e., temporal lobe epilepsy related to mesiotemporal sclerosis (TLE) and extratemporal epilepsy (ETE) related to malformations of cortical development. While these are traditionally conceptualized as 'focal' epilepsies, many patients present with broad structural and functional anomalies. Moreover, the extent of distributed changes contributes to difficulties in multiple cognitive domains as well as affective-behavioral challenges. We will also review work in idiopathic generalized epilepsy (IGE), a class of generalized epilepsy syndromes that involve subcortico-cortical circuits. Overall, neuroimaging and network neuroscience studies point to both shared and syndrome specific connectome signatures of dysfunction across TLE, ETE, and IGE. Lastly, we point to current gaps in the literature and formulate recommendations for future research.
\end{abstract}

KEY WORDS: Epilepsy, cognition, affect, dysfunction, connectomics, neuroimaging, network neuroscience 


\section{INTRODUCTION}

Epilepsy is a neurological disorder characterized by recurrent seizures, affecting around $1 \%$ of the world population (approximately 50 million people) ${ }^{1}$. While seizures can be controlled with anti-seizure medications in most patients, $30-40 \%$ of individuals are drug-resistant, and are at higher risk of widespread and cumulative brain damage ${ }^{2-7}$. Moreover, cognitive impairment has been reported to encompass multiple domains ${ }^{8}$, including memory, executive function, and language abilities. Difficulties in mood and emotion regulation are also increasingly recognized, challenging patient wellbeing and quality of life $9 ; 10$. Collectively, these findings underscore the broad impact of epilepsy on brain health and function and emphasize the importance of shifting our understanding and clinical management of epilepsy as a disorder characterized by more than seizures ${ }^{11}$.

Temporal lobe epilepsy related to mesiotemporal sclerosis (TLE) as well as extratemporal epilepsy related to malformations of cortical development (ETE) are among the most common drug-resistant epilepsy syndromes. While both TLE and ETE have been traditionally labelled as 'focal' epilepsies related to a confined brain region, they are increasingly understood as system-level disorders of interconnected networks ${ }^{12-14}$. A growing body of neuroimaging as well as histopathological findings demonstrates diffuse network anomalies in both syndromes, affecting regions beyond the area responsible for the onset of seizures ${ }^{15-17}$. Moreover, emerging work combining neuroimaging and cognitive testing suggests clear associations between atypical brain connectivity and measures of cognitive as well as affective function. Idiopathic generalized epilepsies (IGE), on the other hand, constitute approximately $20 \%$ of all patients with epilepsy, and include childhood absence epilepsy, juvenile absence epilepsy, juvenile myoclonic epilepsy, and epilepsy with generalized tonic-clonic seizures alone. Neuroimaging studies in IGE have detected widespread anomalies in brain structure, connectivity, and function-paralleling the mounting evidence in TLE and ETE on system-level dysfunction. Furthermore, multiple studies assessing cognitive abilities point to syndrome specific impairments, with for example measurable deficits in executive function as well as social and affective disturbances in patients with juvenile myoclonic epilepsy, and visuo-spatial and language deficits in absence epilepsies ${ }^{18}{ }^{19}$. Collectively, these studies suggest that widespread network anomalies contribute not only to the clinical manifestations of the disease, such as seizure burden and treatment response, but also to cognitive and affective difficulties affecting many patients' lives.

Advances in multimodal neuroimaging and connectome analysis have contributed to our growing understanding of the structural and functional organization of brain networks ${ }^{20}$, and promise to provide candidate metrics that could inform clinical care ${ }^{12}$. In this targeted review, we will survey recent literature assessing connectome biomarkers of cognitive and affective dysfunction across common epilepsy syndromes, notably TLE, ETE, and IGE. Specifically, we will present the current literature on structural and functional network anomalies in each of these syndromes, outline the prevailing notions of syndrome related cognitive dysfunction, and summarize the emerging literature that relates network neuroscience findings to neuropsychological assessments. We will then highlight gaps in the literature and make recommendations for future research on brain and cognition in the common epilepsies.

\section{TEMPORAL LOBE EPILEPSY (TLE)}

The most common and widely studied drug-resistant epilepsy in adults is TLE. This syndrome is traditionally associated with variable degrees of histopathological alterations within mesial temporal lobe structures, such as the hippocampus, amygdala, and entorhinal cortex ${ }^{21-23}$. Mounting histopathological and neuroimaging literature, however, challenges the notion that the condition can be reduced to localized pathology in the mesiotemporal lobes. Histological data suggests that mesiotemporal alterations often cooccur with broad rearrangements of cortical architecture, together with cell loss and gliosis in temporal and extratemporal cortices as well as subcortical structures, particularly the thalamus ${ }^{24 ; 25}$. These findings have been confirmed in vivo, based on structural MRI assessments applying volumetric analysis, as well as with automated surface-based measures ${ }^{15 ; 26-30}$. These studies have reported, in both single and multi-site 
datasets, that TLE is often associated with diffuse cortical atrophy affecting mesiotemporal, lateral temporal, frontal, and centro-parietal cortices, as well as profound atrophy of several subcortical structures $31 ; 32$. While the distribution of grey matter atrophy appears relatively widespread and bilateral, analysis of cortical asymmetries has pointed more directly to marked changes in ipsilateral structures, particularly in temporo-limbic regions. These findings have been complemented by quantitative analysis of other MRI contrasts, such as FLAIR/T2w imaging ${ }^{33}$ as well as quantitative $\mathrm{T} 1$ relaxometry ${ }^{34}$, suggesting that structural alterations are broad, yet nevertheless most concentrated in paralimbic areas ipsilateral to the seizure focus. Similarly, several diffusion MRI studies have described anomalies in structural connectivity and subcortical white matter microstructure, affecting strongly temporo-limbic pathways and with more moderate effect sizes in extralimbic collateral and commissural pathways ${ }^{35-39}$. Analysis of brain function and connectivity, as measured by task-based and resting-state fMRI, has shown atypical functional interactions between temporal and extratemporal areas in TLE patients relative to controls, with findings appearing most marked in temporo-limbic and default mode networks, systems known to be anatomically connected to the mesiotemporal lobe ${ }^{40 ; 41}$. Overall, and despite sometimes diverging topographies of regional changes across different neuroimaging modalities, findings converge towards widespread network compromise in TLE, likely modulated by anatomical connections to a mesiotemporal/paralimbic disease epicenter.

Considering cognitive function, a majority of TLE patients has traditionally been reported to show memory impairment, while around $30-40 \%$ present with difficulties in language function ${ }^{42}{ }^{43-45}$. Paralleling the widespread anomalies revealed by neuroimaging and histology, an increasing body of work suggests that impairments in executive ${ }^{46}$, sensory, and motor functions are also prevalent in TLE ${ }^{10 ; 43-45}$. As such, the landscape of cognitive difficulties in TLE emphasizes a broad profile of impairments across patients affecting multiple cognitive domains. Adding to the complexity of the cognitive dysfunction landscape in TLE, impairments are also quite variable across patients. Several groups have previously suggested the existence of graded abnormalities across TLE patient subgroups, with approximately a third of patients presenting with marked dysfunction while the remaining patients show mild or no measurable impairments compared to age-matched healthy individuals $4748 ; 49$. In TLE, the degree and laterality of structural pathology in the hippocampus have been associated with verbal memory and language impairments ${ }^{50 ; 51}$. Further, functional connectivity measures of the hippocampus and cortical networks have been used to probe language lateralization ${ }^{52-54}$ and to predict deficits in episodic memory ${ }^{55}$. In the latter study, the investigators reported an atypical organization of functional connections that link the hippocampus to wide areas of cortex; the data suggests that the structural reorganization of the hippocampus and its functional interactions with the rest of the brain underpins reorganization of memory networks towards a potentially less efficient functional architecture ${ }^{55}$. Such findings are in line with earlier fMRI findings in TLE patients during memory tasks, showing a reorganization of both ipsilateral and contralateral hippocampal and extrahippocampal functional networks ${ }^{56-59}$. Other work studied intrinsic functional hubs in TLE and reported an association between functional network reorganization and both language and memory impairment in patients ${ }^{60}$. Together, these studies emphasize the role of (i) hippocampal-neocortical subnetworks, and (ii) structural alterations in the mesiotemporal lobes, in determining cognitive deficits in TLE, particularly relating to memory and language function.

A separate and complementary series of studies identified associations between structural connectivity measures and cognitive phenotypes in TLE. There has been robust evidence for an association between diffusion abnormalities of deep white matter fiber tracts as well as the superficial white matter, and reduced language and memory performance in TLE patients ${ }^{61}$. In one study, the authors combined dimensional and categorical multivariate approaches to show that more marked degrees of impairment across several cognitive functions related to overall less efficient and less inter-connected white matter network organization ${ }^{62}$. Another study leveraged communication models, which simulate functional interactions from structural connectivity data, and reported that overall delays in regional interactions related to broad impairments in several cognitive domains ${ }^{63}$. Moving towards a biomarker evaluation framework, 
connectome-based machine learning - with cross-validation and the use of an independent hold-out dataset - has shown utility in predicting memory and language impairment, proving an optimistic outlook that brain network models can aid in indexing patient-specific functional impairments ${ }^{64}{ }^{65}$. In one of these studies, structural connectivity features achieved improved performance when they were combined with hippocampal imaging features, pointing to benefits of combining targeted assessments of the mesiotemporal epicenter with large-scale network models ${ }^{64}$. These studies, thus, emphasize the importance of efficient network communication in preserving cognition in TLE patients, and hold promise in serving as robust biomarkers of cognitive difficulties in this population.

Cognitive dysfunction may co-occur with socio-affective deficits. A substantial proportion of patients suffer from depressive symptoms, anxiety, and personality disorders ${ }^{66 ; 67}$. As for cognitive dysfunction, affective difficulties may have a considerable impact on patient wellbeing and quality of life. In one previous study, the investigators related self-reports from the Beck Depression Inventory to structural and functional connectivity measures in both TLE patients and controls ${ }^{68 ; 69}$. The authors could observe that structural and functional organization of fronto-limbic circuits - in particular connectivity between the hippocampus and anterior frontal regions - reflected depressive symptoms. Fronto-limbic involvement was also suggested by one study relating resting-state fMRI connectivity measures to measures of neuroticism, depression, and anxiety ${ }^{70}$. The authors showed that neuroticism (a personality trait that is associated with depressive symptoms) relates to atypical functional connectivity between mesiotemporal and frontal lobe regions, with connectivity alterations that were partially overlapping to those related to depression and anxiety symptoms ${ }^{71}$. In an earlier study, the authors also reported associations between affective symptoms and reductions in grey matter volumes, again targeting both frontal and temporal neocortices, as well as mesiotemporal lobe structures such as the hippocampus and amygdala ${ }^{72}$. Collectively, previous efforts to study socio-affective functioning in TLE highlight the role of fronto-limbic circuits, as well as the structural integrity of these regions, in mood-related symptoms.

\section{EXTRATEMPORAL LOBE EPILEPSY (ETE)}

Extratemporal lobe epilepsy refers to a broad class of location-related syndromes in which the seizures originate from regions outside of the temporal lobe. The most common ETE subsyndrome is frontal lobe epilepsy, which accounts for approximately $50 \%$ of ETE patients ${ }^{73 ; 74}$. Although lesional etiologies are quite variable in ETE, several patients present with a suspected or histologically confirmed malformation of cortical development, such as focal cortical dysplasia (type-1 or type-2), heterotopia (subcortical nodular, band), or polymicrogyria. Some of these lesions may be subtle and overlooked upon conventional radiological examination, contributing to diagnostic uncertainty and difficulties in offering targeted surgical therapy ${ }^{75 ; 76}$. While significant effort has been invested in characterizing malformations in single patients using structural MRI and, increasingly, connectomics techniques ${ }^{77-81}$, a few recent studies have also characterized the whole-brain substrates of ETE cohorts at a group level. These studies have shown that structural and functional network substrates across different ETE subsyndromes likely vary with respect to the primary lesional etiology. Patients with type-1 focal cortical dysplasia (a late-stage malformation associated with subtle alterations in cortical architecture and intensity) present with widespread cortical thinning relative to controls. In contrast, patients with type- 2 dysplasia (early stage cortical malformations that manifest on MRI as cortical thickening and interface blurring, together with FLAIR/T2w intensity changes ${ }^{82 ; 83}$ ) present with patches of increased cortical thickness beyond the primary lesional perimeter ${ }^{84}$. A previous network analysis of frontal lobe epilepsy patients with different cortical malformations revealed a gradient of functional and structural network anomalies, showing only subtle structural network reorganization in type-2 dysplasia, moderate effects in heterotopias, and maximal changes in late-stage malformations such as type- 1 dysplasia and polymicrogyria ${ }^{78}$. Findings were paralleled by a gradient of functional network anomalies, pointing to reductions in functional network efficiency across the malformation spectrum, again being more pronounced in late-stage cortical malformations such as polymicrogyria compared to early-stage proliferative etiologies ${ }^{85}$. Previous work on ETE thus emphasizes 
the importance of considering the developmental etiology of lesional subtypes to understand structural and functional network anomalies in specific patient subgroups.

In ETE, the landscape of cognitive impairment is less well characterized than in TLE, owing to heterogeneity of the patients' primary etiology, and variable extent of the epileptogenic network. Findings nevertheless suggest an important modulation of impairments with respect to the location of the disease epicenter. For instance, ETE patients with a suspected or confirmed anomaly in the frontal lobes will often present with executive dysfunction ${ }^{86-90}$, ranging from impaired attention to difficulties in goal-oriented behaviors. Verbal competencies also appear impaired ${ }^{91 ; 92}$, and reports have also pointed to poorer motor coordination and reduced psychomotor speed ${ }^{87 ; 93}$. Patients with primary lesions in central and posterior regions may show deficits in visuo-spatial functions, often together with attentional and motor dysfunction. Similar heterogeneity exists even within subgroups of patients with the same lesional subtype, where deficits can range from no apparent cognitive impairment to marked disability.

Studies assessing brain substrates of cognitive deficits in patients with ETE are less frequent than in TLE, and are also more difficult to aggregate due to the above-mentioned heterogeneity across the patient spectrum. However, prior work in ETE cohorts (sometimes mixed with TLE) reported structural and functional connectivity alterations that relate to overall reductions in cognitive function ${ }^{94 ; 95}$. In mixed focal epilepsy cohorts including patients with ETE, prior work highlighted fronto-temporal connectivity abnormalities and global alterations of functional network architecture during language tasks ${ }^{96 ; 97}$, and prefrontal connectional rearrangements during verbal working memory ${ }^{98}$. In children with frontal lobe epilepsy, recent studies showed altered functional connectivity among fronto-temporo-parietal cortices during a working memory task compared to controls ${ }^{99}$, and also reported an association between atypical functional network modularity, consisting of reduced long-range connectivity and increased short-range connections, and reductions in cognitive performance ${ }^{94}$. Notably, associations between cognitive impairment and network topology have been reported in structure as well as function. For example, structural networks estimated from diffusion MRI tractography evince marked alterations in segregation and integration in patients with more severe cognitive impairment ${ }^{95}$; similarly, increased clustering in structural covariance networks derived from T1-weighted data was not only associated with markers of disease severity, but also with lower general intelligence ${ }^{100}$. In a recent study, we combined a comprehensive cognitive profiling of language and working memory abilities with task-based fMRI analysis and connectome-level contextualization in a cohort with frontal lobe epilepsy, and compared findings to both patients with TLE and healthy controls ${ }^{101}$. Our study showed that working memory and language impairment in frontal lobe epilepsy was associated with reduced activation across attentional and executive systems, together with an attenuated deactivation of default mode regions, suggesting a reorganization of functional recruitment at the systems level. While atypical activation patterns were similar to those in TLE patients, reductions in default mode network deactivations appeared more marked in frontal lobe epilepsy patients, whereas those with TLE presented with less activations in posterior language areas during semantic tasks ${ }^{101}$. Collectively, these findings provide emerging evidence of both shared and syndrome specific impacts of different location-related epilepsies on brain functional networks. Moreover, this work broadly illustrates the utility of network neuroscience approaches in capturing important substrates that contribute to inter-patient variability in cognitive dysfunction.

Studies of socio-affective functioning are less common than studies of cognitive function in ETE, but represent an important and growing area of research. One previous study in a cohort of 40 frontal lobe epilepsy cases reported that $25 \%$ have elevated anxiety scores, $40 \%$ have either elevated depression or elevated anxiety scores, and $25 \%$ have depression scores above the clinical cut-off ${ }^{102}$. These values appear higher than in patients with generalized epilepsies ${ }^{102}$, but are not as high as those observed in TLE ${ }^{103}$. Further, prior work highlights that executive dysfunction in frontal lobe epilepsy can co-occur with reduced response inhibition, hyperactivity, obsession, and addictive behaviors that may interfere with overall adaptation to everyday life ${ }^{103}$. While there is abundant literature on behavioral and emotional difficulties 
in patients with specific lesions, including foundational case studies in patients with frontal lobe lesions, there are, to our knowledge, no systematic assessments of the relationship between whole-brain connectome architecture and affective difficulties in ETE patients.

\section{IDIOPATHIC/GENETIC GENERALIZED EPILEPSY (IGE)}

Idiopathic generalized epilepsy refers to a group of epilepsy syndromes with likely polygenetic inheritance, and characterized by generalized spike and slow-wave discharges on EEG. While multiple syndromes are subsumed under the umbrella term of IGE ${ }^{104-106}$, converging evidence supports a key role of thalamocortical networks ${ }^{107}$. Fronto-thalamo-cortical involvement during generation and propagation of generalized seizures has been solidified by electrophysiological work ${ }^{108 ;}{ }^{109}$. Structural MRI studies complemented these findings, by indicating grey matter volume reductions in both the thalamus and neocortex ${ }^{110}$, albeit to a lesser extent than in syndromes such as TLE ${ }^{31 ; 111}$. In addition to atrophy, recent work also pointed to atypical cortical folding and cortex-cortical distance relationships in juvenile myoclonic epilepsy, with the latter also being present in non-affected siblings ${ }^{112}$. Atypical cortical folding may signify perturbations in underlying brain connections; resting-state fMRI approaches have indeed supported atypical organization of cortico-thalamic networks in different IGE syndromes, showing aberrant connectivity when seeding from either cortical or thalamic regions ${ }^{113-115}$. Similar reports have appeared in diffusion MRI studies, which showed atypical structural connectivity between thalamic and medial frontal regions in IGE subgroups with juvenile myoclonic epilepsy ${ }^{116}$.

The cognitive landscape of IGE is also characterized by atypical function across several domains. A systematic review and meta-analysis conducted across 26 studies revealed broad reductions in cognitive function across multiple domains in IGE patients, involving generalized cognitive ability, fluid/crystallized intelligence, processing speed, and memory abilities ${ }^{117}$. Impairments were observed in studies assessing mixed IGE cohorts, but also when considering specific IGE subsyndromes such as juvenile myoclonic, childhood absence epilepsies, as well as epilepsy with generalized tonic-clonic seizures alone. Executive dysfunction represent a common trait across IGE subsyndromes ${ }^{18}$, pointing to frontal lobe involvement, while impairment of visuospatial abilities may generally be subtle ${ }^{117}$. Another review focusing on childhood absence epilepsy also showed reductions in verbal skills across language and learning tasks, as well as mild impairments in executive functions ${ }^{118}$. In juvenile myoclonic epilepsy, several studies also point to impaired social cognition ${ }^{18 ; 19}$, which may possibly relate to the consistent frontal lobe dysfunction seen in the disorder. Profiles of cognitive difficulties in IGE are thus seemingly broad, with specific subsyndromes possibly showing more circumscribed patterns of impairment.

Taken together, studies using cognitive and neuroimaging measures have supported an overall perturbation in the organization of prefronto-central-thalamic connections. A positron emission tomography study reported prefrontal and subcortical glucose hypometabolism in patients with juvenile myoclonic epilepsy, and associations with reduced working memory and mental flexibility ${ }^{119}$. These findings are complemented by task fMRI studies in the same IGE subsyndrome, which report altered motor activation across a battery of cognitive tasks ${ }^{120 ; 121}$, and atypical connectivity between prefrontal and motor networks during a working memory paradigm, ${ }^{121}$ which may constitute heritable traits ${ }^{120 ; 122}$. Atypical functional connectivity between prefrontal and thalamic regions was also observed during a verbal fluency task ${ }^{116}$, and was shown to relate to both reduced verbal fluency and decremented structural connectivity between these regions. Other structural and diffusion MRI studies also support a network basis for impairment, by underscoring associations between grey and white matter alterations in both cortical and thalamic regions and cognitive impairments in mental flexibility, language, and memory function ${ }^{123-125}$. Compared to the broad literature on network correlates of dysfunction in juvenile myoclonic epilepsy, association studies between imaging measures and cognitive variables are less frequent in absence epilepsy ${ }^{18}$. One study reported atypical cortical morphology and folding in frontal and temporal cortices, and indirectly supported a potential contribution to reductions in verbal and performance IQ measures ${ }^{126}$. During a sustained attention paradigm, a functional imaging study revealed an association between lower activation of the medial frontal 
cortex and impaired performance, which co-existed with reduction of fronto-insular resting-state connectivity ${ }^{127}$. Collectively, although the amount of work associating cognitive phenotypes with brain network information is at times more limited for certain IGE subsyndromes, the literature overall suggests that atypical wiring and cross-talk of thalamic and cortical regions may contribute to the sometimes rather broad impairments seen in IGE patients.

\section{CONCLUSIONS AND CURRENT GAPS}

Neuroimaging and connectomics approaches are beginning to reveal structural, functional, and network level substrates in TLE, ETE, and IGE. These studies support both shared and syndrome specific patterns of cortico-subcortical reorganization across different epilepsy syndromes, with TLE being associated with marked and widespread network reorganization that is particularly extensive in the proximity of the mesiotemporal epicenter. While memory and language deficits are most commonly reported cognitive difficulties, mounting evidence suggests that white matter microstructure and functional alterations also contribute to dysfunction in other cognitive domains, such as executive function. Fronto-limbic alterations in brain structure and function also contribute to commonly observed affective comorbidities, including depression and anxiety, and may potentially underpin other traits associated with TLE, such as neuroticism. In ETE, neuroimaging and connectomics studies suggest widespread impairments in structural and functional brain network organization, which seems to be modulated by both location and etiology of the primary lesion. For example, ETE patients with a suspected or confirmed anomaly in frontal circuits will often present with executive dysfunction as well as reduced verbal competence, sometimes together with poorer motor coordination and reduced psychomotor speed. Emerging literature has furthermore begun to reveal structural and functional substrates of cognitive impairments in ETE, suggesting reorganization in multiple brain subnetworks, often characterized by atypical activation/deactivation patterns of large-scale functional systems. Finally, although the different syndromes subsumed under the IGE umbrella term are relatively heterogenous, consistent structural and functional imaging findings point to thalamo-cortical structural and functional dysfunction. Across different IGE subsyndromes, most work has been performed in juvenile myoclonic epilepsy, pointing to similar, albeit somewhat more subtle cognitive impairment than in ETE patients with a frontal lobe seizure focus.

Epilepsy is truly more than a seizure condition, and it is thus important to continue to identify underlying mechanisms of cognitive function across the spectrum of common epilepsies. This expansion will involve increasing research efforts into less prevalent syndromes and further studying sources of inter-patient variability. For these studies, we emphasize the need to combine multidimensional cognitive phenotyping approaches with multimodal neuroimaging, given the likely complementary power of structural and functional imaging techniques. In TLE, prior clustering and multivariate associative studies are already beginning to shed light on covariations in atypical connectome organization and cognition, and further help to clarify sources of inter-individual variations across the patient spectrum. Paralleling current trends in the study of the brain and mental health, we recommend cross-syndrome investigations that can identify shared and syndrome specific effects on brain network organization and cognition. Such approaches will provide higher granularity in studying the panorama of cognitive and affective impairments and associated connectome anomalies in TLE, ETE, and IGE. Finally, to generalize from potential idiosyncrasies of specific epilepsy centres and to allow for an unbiased evaluation of network biomarkers in the prediction of dysfunction at the single-patient level, we recommend multi-site data aggregation and analysis efforts

such as ENIGMA-Epilepsy, ${ }^{128}$ along with strategies for prospective as well as retrospective data harmonization at the level of brain and cognition alike.

\section{ACKNOWLEDGEMENTS}

RRC acknowledges research support from the Fonds de la Recherche du Québec - Santé (FRQ-S 291486). JR is supported by a Fellowship from the Canadian Institute of Health Research (CIHR). BCB acknowledges research support from the National Science and Engineering Research Council of Canada (NSERC Discovery-1304413), the CIHR (FDN-154298, PJT-174995), SickKids Foundation (NI17-039), 
Azrieli Center for Autism Research (ACAR-TACC), BrainCanada, FRQ-S, and the Tier2 Canada Research Chairs program. SL is supported by a CIHR doctoral award. B.C.B acknowledges research support from the National Science and Engineering Research Council of Canada (NSERC Discovery-1304413), the CIHR (FDN-154298, PJT-174995), SickKids Foundation (NI17-039), Azrieli Center for Autism Research (ACAR-TACC), BrainCanada, FRQ-S, the Helmholtz International Analytics Learning Laboratory (Hiball), and the Tier-2 Canada Research Chairs program. L.C. and D.S.B. acknowledge support from the NINDS (R01-NS099348). D.S.B. acknowledges support from the John D. and Catherine T. MacArthur Foundation, the Alfred P. Sloan Foundation, the Paul Allen Family Foundation, and the ISI Foundation. 


\section{REFERENCES}

1. Collaborators GBDN. Global, regional, and national burden of neurological disorders, 1990-2016: a systematic analysis for the Global Burden of Disease Study 2016. Lancet Neurol 2019;18:459-480.

2. Caciagli L, Bernasconi A, Wiebe S, et al. Time is brain? A meta-analysis on progressive atrophy in intractable temporal lobe epilepsy. Neurology 2017;89(5):506-516.

3. Bernhardt BC, Kim H, Bernasconi N. Patterns of subregional mesiotemporal disease progression in temporal lobe epilepsy. Neurology 2013;81:1840-1847.

4. Bernasconi N, Bernhardt BC. Temporal lobe epilepsy is a progressive disorder. Nat Rev Neurol 2010;6:1.

5. Bernhardt $\mathrm{BC}$, Worsley KJ, Kim H, et al. Longitudinal and cross-sectional analysis of atrophy in pharmacoresistant temporal lobe epilepsy. Neurology 2009;72:1747-1754.

6. Coan AC, Appenzeller S, Bonilha L, et al. Seizure frequency and lateralization affect progression of atrophy in temporal lobe epilepsy. Neurology 2009;73:834-842.

7. Galovic M, Baudracco I, Wright-Goff E, et al. Association of Piriform Cortex Resection With Surgical Outcomes in Patients With Temporal Lobe Epilepsy. JAMA Neurol 2019;76:690-700.

8. Loring DW, Meador KJ, Lee GP. Determinants of quality of life in epilepsy. Epilepsy \& behavior : E\&B 2004;5:976-980.

9. Bell B, Lin JJ, Seidenberg M, et al. The neurobiology of cognitive disorders in temporal lobe epilepsy. Nat Rev Neurol 2011;7:154-164.

10. Hermann BP, Lin JJ, Jones JE, et al. The emerging architecture of neuropsychological impairment in epilepsy. Neurol Clin 2009;27:881-907.

11. Selassie AW, Wilson DA, Martz GU, et al. Epilepsy beyond seizure: a population-based study of comorbidities. Epilepsy research 2014;108:305-315.

12. Lariviere S, Bernasconi A, Bernasconi N, et al. Connectome biomarkers of drug-resistant epilepsy. Epilepsia 2021;62:6-24.

13. Tavakol S, Royer J, Lowe AJ, et al. Neuroimaging and connectomics of drug-resistant epilepsy at multiple scales: From focal lesions to macroscale networks. Epilepsia 2019;60:593-604.

14. Gleichgerrcht E, Kocher M, Bonilha L. Connectomics and graph theory analyses: Novel insights into network abnormalities in epilepsy. Epilepsia 2015;56:1660-1668.

15. Bernhardt BC, Hong SJ, Bernasconi A, et al. Magnetic resonance imaging pattern learning in temporal lobe epilepsy: Classification and prognostics. Ann Neurol 2015;77:436-446.

16. Caciagli L, Bernhardt BC, Hong SJ, et al. Functional network alterations and their structural substrate in drug-resistant epilepsy. Front Neurosci 2014;8:411.

17. Bernhardt $\mathrm{BC}$, Hong $\mathrm{S}$, Bernasconi $\mathrm{A}$, et al. Imaging structural and functional brain networks in temporal lobe epilepsy. Front Hum Neurosci 2013;7:624.

18. Ratcliffe C, Wandschneider B, Baxendale S, et al. Cognitive Function in Genetic Generalized Epilepsies: Insights From Neuropsychology and Neuroimaging. Frontiers in neurology 2020;11:144.

19. Guida M, Caciagli L, Cosottini M, et al. Social cognition in idiopathic generalized epilepsies and potential neuroanatomical correlates. Epilepsy \& behavior : E\&B 2019;100:106118.

20. Lariviere S, Vos de Wael R, Paquola C, et al. Microstructure-informed connectomics: enriching largescale descriptions of healthy and diseased brains. Brain connectivity 2018.

21. Blumcke I, Thom M, Aronica E, et al. International consensus classification of hippocampal sclerosis in temporal lobe epilepsy: A Task Force report from the ILAE Commission on Diagnostic Methods. Epilepsia 2013;54:1315-1329.

22. Thom M. Review: Hippocampal sclerosis in epilepsy: a neuropathology review. Neuropathol Appl Neurobiol 2014;40:520-543.

23. Thom M, Eriksson S, Martinian L, et al. Temporal lobe sclerosis associated with hippocampal sclerosis in temporal lobe epilepsy: neuropathological features. J Neuropathol Exp Neurol 2009;68:928-938.

24. Blanc F, Martinian L, Liagkouras I, et al. Investigation of widespread neocortical pathology associated with hippocampal sclerosis in epilepsy: a postmortem study. Epilepsia 2011;52:10-21.

25. Margerison JH, Corsellis JA. Epilepsy and the temporal lobes. A clinical, electroencephalographic and 
neuropathological study of the brain in epilepsy, with particular reference to the temporal lobes. Brain 1966;89:499-530.

26. Lin JJ, Salamon N, Lee AD, et al. Reduced neocortical thickness and complexity mapped in mesial temporal lobe epilepsy with hippocampal sclerosis. Cereb Cortex 2007;17:2007-2018.

27. McDonald CR, Hagler DJ, Jr., Ahmadi ME, et al. Regional neocortical thinning in mesial temporal lobe epilepsy. Epilepsia 2008;49:794-803.

28. Bernhardt BC, Bernasconi A, Liu M, et al. The spectrum of structural and functional imaging abnormalities in temporal lobe epilepsy. Annals of neurology 2016;80:142-153.

29. Bernhardt BC, Bernasconi N, Kim H, et al. Mapping thalamocortical network pathology in temporal lobe epilepsy. Neurology 2012;78:129-136.

30. Bernhardt BC, Worsley KJ, Besson P, et al. Mapping limbic network organization in temporal lobe epilepsy using morphometric correlations: insights on the relation between mesiotemporal connectivity and cortical atrophy. Neuroimage 2008;42:515-524.

31. Whelan CD, Altmann A, Botia JA, et al. Structural brain abnormalities in the common epilepsies assessed in a worldwide ENIGMA study. Brain : a journal of neurology 2018;141:391-408.

32. Lariviere S, Rodriguez-Cruces R, Royer J, et al. Network-based atrophy modeling in the common epilepsies: A worldwide ENIGMA study. Sci Adv 2020;6.

33. Adler S, Hong SJ, Liu M, et al. Topographic principles of cortical fluid-attenuated inversion recovery signal in temporal lobe epilepsy. Epilepsia 2018;59:627-635.

34. Bernhardt BC, Fadaie F, Vos de Wael R, et al. Preferential susceptibility of limbic cortices to microstructural damage in temporal lobe epilepsy: A quantitative T1 mapping study. NeuroImage 2018;182:294-303.

35. Concha L, Kim H, Bernasconi A, et al. Spatial patterns of water diffusion along white matter tracts in temporal lobe epilepsy. Neurology 2012;79:455-462.

36. Concha L, Beaulieu C, Collins DL, et al. White-matter diffusion abnormalities in temporal-lobe epilepsy with and without mesial temporal sclerosis. J Neurol Neurosurg Psychiatry 2009;80:312-319.

37. Concha L, Beaulieu C, Gross DW. Bilateral limbic diffusion abnormalities in unilateral temporal lobe epilepsy. Ann Neurol 2005;57:188-196.

38. Focke NK, Yogarajah M, Bonelli SB, et al. Voxel-based diffusion tensor imaging in patients with mesial temporal lobe epilepsy and hippocampal sclerosis. Neuroimage 2008;40:728-737.

39. Liu M, Bernhardt BC, Hong SJ, et al. The superficial white matter in temporal lobe epilepsy: a key link between structural and functional network disruptions. Brain 2016;139:2431-2440.

40. Bernhardt $\mathrm{BC}$, Bernasconi $\mathrm{A}$, Liu $\mathrm{M}$, et al. The spectrum of structural and functional imaging abnormalities in temporal lobe epilepsy. Ann Neurol 2016;80:142-153.

41. Lariviere S, Weng Y, Vos de Wael R, et al. Functional connectome contractions in temporal lobe epilepsy: Microstructural underpinnings and predictors of surgical outcome. Epilepsia 2020.

42. Reyes A, Holden HM, Chang YA, et al. Impaired spatial pattern separation performance in temporal lobe epilepsy is associated with visuospatial memory deficits and hippocampal volume loss. Neuropsychologia 2018;111:209-215.

43. Lin JJ, Mula M, Hermann BP. Uncovering the neurobehavioural comorbidities of epilepsy over the lifespan. Lancet 2012;380:1180-1192.

44. McAndrews MP, Cohn M. Neuropsychology in temporal lobe epilepsy: influences from cognitive neuroscience and functional neuroimaging. Epilepsy research and treatment 2012;2012:925238.

45. Saling MM. Verbal memory in mesial temporal lobe epilepsy: beyond material specificity. Brain : $a$ journal of neurology 2009;132:570-582.

46. Lutz MT, Helmstaedter C. EpiTrack: tracking cognitive side effects of medication on attention and executive functions in patients with epilepsy. Epilepsy Behav 2005;7:708-714.

47. Rodriguez-Cruces R, Velazquez-Perez L, Rodriguez-Leyva I, et al. Association of white matter diffusion characteristics and cognitive deficits in temporal lobe epilepsy. Epilepsy \& behavior : E\&B 2018;79:138-145.

48. Hermann B, Seidenberg M, Lee EJ, et al. Cognitive phenotypes in temporal lobe epilepsy. Journal of 
the International Neuropsychological Society: JINS 2007;13:12-20.

49. Reyes A, Kaestner E, Ferguson L, et al. Cognitive phenotypes in temporal lobe epilepsy utilizing dataand clinically driven approaches: Moving toward a new taxonomy. Epilepsia 2020;61:1211-1220.

50. Stewart CC, Griffith HR, Okonkwo OC, et al. Contributions of volumetrics of the hippocampus and thalamus to verbal memory in temporal lobe epilepsy patients. Brain Cogn 2009;69:65-72.

51. Dabbs K, Jones J, Seidenberg M, et al. Neuroanatomical correlates of cognitive phenotypes in temporal lobe epilepsy. Epilepsy Behav 2009;15:445-451.

52. Lopes TM, de Campos BM, Zanao TA, et al. Hippocampal atrophy disrupts the language network but not hemispheric language lateralization. Epilepsia 2019;60:744-755.

53. Berl MM, Zimmaro LA, Khan OI, et al. Characterization of atypical language activation patterns in focal epilepsy. Ann Neurol 2013.

54. Benjamin CF, Walshaw PD, Hale K, et al. Presurgical language fMRI: Mapping of six critical regions. Human brain mapping 2017;38:4239-4255.

55. Li Q, Tavakol S, Royer J, et al. Atypical neural topographies underpin dysfunctional pattern separation in temporal lobe epilepsy. Brain : a journal of neurology 2021;in press.

56. Sidhu MK, Stretton J, Winston GP, et al. A functional magnetic resonance imaging study mapping the episodic memory encoding network in temporal lobe epilepsy. Brain 2013;136:1868-1888.

57. Jokeit H, Okujava M, Woermann FG. Memory fMRI lateralizes temporal lobe epilepsy. Neurology 2001;57:1786-1793.

58. Bigras C, Shear PK, Vannest J, et al. The effects of temporal lobe epilepsy on scene encoding. Epilepsy \& behavior : $E \& B$ 2013;26:11-21.

59. Alessio A, Pereira FR, Sercheli MS, et al. Brain plasticity for verbal and visual memories in patients with mesial temporal lobe epilepsy and hippocampal sclerosis: an fMRI study. Human brain mapping 2013;34:186-199.

60. Roger E, Pichat C, Torlay L, et al. Hubs disruption in mesial temporal lobe epilepsy. A resting-state fMRI study on a language-and-memory network. Human brain mapping 2020;41:779-796.

61. Reyes A, Kaestner E, Bahrami N, et al. Cognitive phenotypes in temporal lobe epilepsy are associated with distinct patterns of white matter network abnormalities. Neurology 2019;92:e1957-e1968.

62. Rodriguez-Cruces R, Bernhardt BC, Concha L. Multidimensional associations between cognition and connectome organization in temporal lobe epilepsy. NeuroImage 2019;213.

63. Girardi-Schappo M, Fadaie F, Lee HM, et al. Altered communication dynamics reflect cognitive deficits in temporal lobe epilepsy. Epilepsia 2021;62:1022-1033.

64. Balachandra AR, Kaestner E, Bahrami N, et al. Clinical utility of structural connectomics in predicting memory in temporal lobe epilepsy. Neurology 2020;94:e2424-e2435.

65. Kaestner E, Balachandra AR, Bahrami N, et al. The white matter connectome as an individualized biomarker of language impairment in temporal lobe epilepsy. NeuroImage. Clinical 2020;25:102125.

66. Tellez-Zenteno JF, Patten SB, Jette N, et al. Psychiatric comorbidity in epilepsy: a population-based analysis. Epilepsia 2007;48:2336-2344.

67. Kanner AM, Schachter SC, Barry JJ, et al. Depression and epilepsy: epidemiologic and neurobiologic perspectives that may explain their high comorbid occurrence. Epilepsy \& behavior : E\&B 2012;24:156-168.

68. Chen S, Wu X, Lui S, et al. Resting-state fMRI study of treatment-naive temporal lobe epilepsy patients with depressive symptoms. Neuroimage 2012;60:299-304.

69. Kemmotsu N, Kucukboyaci NE, Cheng CE, et al. Alterations in functional connectivity between the hippocampus and prefrontal cortex as a correlate of depressive symptoms in temporal lobe epilepsy. Epilepsy Behav 2013;29:552-559.

70. Doucet GE, Skidmore C, Sharan AD, et al. Functional connectivity abnormalities vary by amygdala subdivision and are associated with psychiatric symptoms in unilateral temporal epilepsy. Brain and cognition 2013;83:171-182.

71. Rivera Bonet CN, Hwang G, Hermann B, et al. Neuroticism in temporal lobe epilepsy is associated 
with altered limbic-frontal lobe resting-state functional connectivity. Epilepsy \& behavior : E\&B 2020;110:107172.

72. Rivera Bonet CN, Hermann B, Cook CJ, et al. Neuroanatomical correlates of personality traits in temporal lobe epilepsy: Findings from the Epilepsy Connectome Project. Epilepsy \& behavior : $E \& B$ 2019;98:220-227.

73. Lee JJ, Lee SK, Lee SY, et al. Frontal lobe epilepsy: clinical characteristics, surgical outcomes and diagnostic modalities. Seizure : the journal of the British Epilepsy Association 2008;17:514-523.

74. Delev D, Oehl B, Steinhoff BJ, et al. Surgical Treatment of Extratemporal Epilepsy: Results and Prognostic Factors. Neurosurgery 2019;84:242-252.

75. Bernasconi A, Bernasconi N, Bernhardt BC, et al. Advances in MRI for 'cryptogenic' epilepsies. Nat Rev Neurol 2011;7:99-108.

76. Bernasconi A, Bernasconi N. Unveiling epileptogenic lesions: the contribution of image processing. Epilepsia 2011;52 Suppl 4:20-24.

77. Hong S, Bernhardt BC, Gill R, et al. Connectome-Based Pattern Learning Predicts Histology and Surgical Outcome of Epileptogenic Malformations of Cortical Development. MICCAI 2017:390397.

78. Hong S, Bernhardt BC, Gill RS, et al. The spectrum of structural and functional network anomalies across the spectrum of malformations of cortical development. Brain : a journal of neurology 2017; 140:2133-2143.

79. Bernasconi A, Antel SB, Collins DL, et al. Texture analysis and morphological processing of magnetic resonance imaging assist detection of focal cortical dysplasia in extra-temporal partial epilepsy. Ann Neurol 2001;49:770-775.

80. Colliot $\mathrm{O}$, Antel $\mathrm{S}$, Naessens $\mathrm{V}$, et al. In vivo profiling of focal cortical dysplasia on high-resolution MRI using computational models. Epilepsia 2005:(submitted).

81. Wang ZI, Jones SE, Jaisani Z, et al. Voxel-based morphometric magnetic resonance imaging (MRI) postprocessing in MRI-negative epilepsies. Ann Neurol 2015;77:1060-1075.

82. Hong S, Kim H, Bernasconi N, et al. Automated detection of cortical dysplasia type II in MRI-negative epilepsy. Neurology 2014;83:48-55.

83. Hong SJ, Bernhardt B, Schrader D, et al. MRI-Based Lesion Profiling of Epileptogenic Cortical Malformations. Medical Image Computing and Computer-Assisted Intervention - MICCAI 2015;9350:501-509.

84. Hong S, Bernhardt BC, Schrader DV, et al. Whole-brain MRI phenotying of dysplasia-related frontal lobe epilepsy. Neurology 2016;86:643-650.

85. Hong S-J, Bernhardt BC, Gill RS, et al. The spectrum of structural and functional network alterations in malformations of cortical development. Brain : a journal of neurology 2017;140:2133-2143.

86. Verche E, San Luis C, Hernandez S. Neuropsychology of frontal lobe epilepsy in children and adults: Systematic review and meta-analysis. Epilepsy Behav 2018;88:15-20.

87. Upton D, Thompson PJ. General neuropsychological characteristics of frontal lobe epilepsy. Epilepsy research 1996;23:169-177.

88. Exner C, Boucsein K, Lange C, et al. Neuropsychological performance in frontal lobe epilepsy. Seizure : the journal of the British Epilepsy Association 2002;11:20-32.

89. Patrikelis P, Gatzonis S, Siatouni A, et al. Preoperative neuropsychological presentation of patients with refractory frontal lobe epilepsy. Acta neurochirurgica 2016;158:1139-1150.

90. Braakman HM, Vaessen MJ, Hofman PA, et al. Cognitive and behavioral complications of frontal lobe epilepsy in children: a review of the literature. Epilepsia 2011;52:849-856.

91. Risse GL. Cognitive outcomes in patients with frontal lobe epilepsy. Epilepsia 2006;47 Suppl 2:87-89.

92. Helmstaedter C, Kemper B, Elger CE. Neuropsychological aspects of frontal lobe epilepsy. Neuropsychologia 1996;34:399-406.

93. Upton D, Thompson PJ. Neuropsychological test performance in frontal-lobe epilepsy: the influence of aetiology, seizure type, seizure frequency and duration of disorder. Seizure : the journal of the British Epilepsy Association 1997;6:443-447. 
94. Vaessen MJ, Braakman HM, Heerink JS, et al. Abnormal modular organization of functional networks in cognitively impaired children with frontal lobe epilepsy. Cereb Cortex 2013;23:1997-2006.

95. Vaessen MJ, Jansen JF, Vlooswijk MC, et al. White matter network abnormalities are associated with cognitive decline in chronic epilepsy. Cereb Cortex 2012;22:2139-2147.

96. Vlooswijk MC, Vaessen MJ, Jansen JF, et al. Loss of network efficiency associated with cognitive decline in chronic epilepsy. Neurology 2011;77:938-944.

97. Vlooswijk MC, Jansen JF, Majoie HJ, et al. Functional connectivity and language impairment in cryptogenic localization-related epilepsy. Neurology 2010;75:395-402.

98. Vlooswijk MC, Jansen JF, Jeukens CR, et al. Memory processes and prefrontal network dysfunction in cryptogenic epilepsy. Epilepsia 2011;52:1467-1475.

99. Braakman HM, Vaessen MJ, Jansen JF, et al. Frontal lobe connectivity and cognitive impairment in pediatric frontal lobe epilepsy. Epilepsia 2013;54:446-454.

100. Drenthen GS, Backes WH, Rouhl RPW, et al. Structural covariance networks relate to the severity of epilepsy with focal-onset seizures. NeuroImage. Clinical 2018;20:861-867.

101. Caciagli L, Paquola C, He X, et al. Disorganization of language and working memory systems in frontal versus temporal lobe epilepsy. bioarxiv 2021.

102. Tang WK, Lu J, Ungvari GS, et al. Anxiety symptoms in patients with frontal lobe epilepsy versus generalized epilepsy. Seizure : the journal of the British Epilepsy Association 2012;21:457-460.

103. Helmstaedter C. Behavioral Aspects of Frontal Lobe Epilepsy. Epilepsy Behav 2001;2:384-395.

104. Nordli DR, Jr. Idiopathic generalized epilepsies recognized by the International League Against Epilepsy. Epilepsia 2005;46 Suppl 9:48-56.

105. ILAE. Commission on Classification and Terminology of the International League Against Epilepsy: Proposal for classification of epilepsies and epileptic syndromes. Epilepsia 1989;30:389-399.

106. Andermann F, Berkovic SF. Idiopathic generalized epilepsy with generalized and other seizures in adolescence. Epilepsia 2001;42:317-320.

107. Kwan P, Schachter SC, Brodie MJ. Drug-resistant epilepsy. The New England journal of medicine 2011;365:919-926.

108. Blumenfeld H. From molecules to networks: cortical/subcortical interactions in the pathophysiology of idiopathic generalized epilepsy. Epilepsia 2003;44 Suppl 2:7-15.

109. Gotman J, Grova C, Bagshaw A, et al. Generalized epileptic discharges show thalamocortical activation and suspension of the default state of the brain. Proceedings of the National Academy of Sciences of the United States of America 2005;102:15236-15240.

110. Bernhardt BC, Rozen DA, Worsley KJ, et al. Thalamo-cortical network pathology in idiopathic generalized epilepsy: Insights from MRI-based morphometric correlation analysis. NeuroImage 2009;46:373-381.

111. Weng Y, Lariviere S, Caciagli L, et al. Macroscale and microcircuit dissociation of focal and generalized human epilepsies. Commun Biol 2020;3:244.

112. Wandschneider B, Hong SJ, Bernhardt BC, et al. Developmental MRI markers cosegregate juvenile patients with myoclonic epilepsy and their healthy siblings. Neurology 2019;93:e1272-e1280.

113. Wang Z, Zhang Z, Jiao Q, et al. Impairments of thalamic nuclei in idiopathic generalized epilepsy revealed by a study combining morphological and functional connectivity MRI. PloS one 2012;7:e39701.

114. Wang Z, Lu G, Zhang Z, et al. Altered resting state networks in epileptic patients with generalized tonic-clonic seizures. Brain Res 2011;1374:134-141.

115. Wang Z, Lariviere S, Xu Q, et al. Community-informed connectomics of the thalamo-cortical system in generalized epilepsy. Neurology 2019;93.

116. O'Muircheartaigh J, Vollmar C, Barker GJ, et al. Abnormal thalamocortical structural and functional connectivity in juvenile myoclonic epilepsy. Brain 2012;135:3635-3644.

117. Loughman A, Bowden SC, D'Souza W. Cognitive functioning in idiopathic generalised epilepsies: a systematic review and meta-analysis. Neurosci Biobehav Rev 2014;43:20-34.

118. Verrotti A, Matricardi S, Rinaldi VE, et al. Neuropsychological impairment in childhood absence 
epilepsy: Review of the literature. Journal of the neurological sciences 2015;359:59-66.

119. McDonald CR, Swartz BE, Halgren E, et al. The relationship of regional frontal hypometabolism to executive function: a resting fluorodeoxyglucose PET study of patients with epilepsy and healthy controls. Epilepsy \& behavior : E\&B 2006;9:58-67.

120. Caciagli L, Wandschneider B, Centeno M, et al. Motor hyperactivation during cognitive tasks: An endophenotype of juvenile myoclonic epilepsy. Epilepsia 2020;61:1438-1452.

121. Vollmar C, O'Muircheartaigh J, Barker GJ, et al. Motor system hyperconnectivity in juvenile myoclonic epilepsy: a cognitive functional magnetic resonance imaging study. Brain : a journal of neurology 2011;134:1710-1719.

122. Wandschneider B, Centeno M, Vollmar C, et al. Motor co-activation in siblings of patients with juvenile myoclonic epilepsy: an imaging endophenotype? Brain : a journal of neurology 2014;137:2469-2479.

123. O'Muircheartaigh J, Vollmar C, Barker GJ, et al. Focal structural changes and cognitive dysfunction in juvenile myoclonic epilepsy. Neurology 2011;76:34-40.

124. Caeyenberghs K, Powell HW, Thomas RH, et al. Hyperconnectivity in juvenile myoclonic epilepsy: a network analysis. NeuroImage. Clinical 2015;7:98-104.

125. Caciagli L, Wandschneider B, Xiao F, et al. Abnormal hippocampal structure and function in juvenile myoclonic epilepsy and unaffected siblings. Brain : a journal of neurology 2019;142:2670-2687.

126. Tosun D, Siddarth P, Toga AW, et al. Effects of childhood absence epilepsy on associations between regional cortical morphometry and aging and cognitive abilities. Human brain mapping 2011;32:580-591.

127. Killory BD, Bai X, Negishi M, et al. Impaired attention and network connectivity in childhood absence epilepsy. NeuroImage 2011;56:2209-2217.

128. Sisodiya SM, Whelan CD, Hatton SN, et al. The ENIGMA-Epilepsy working group: Mapping disease from large data sets. Human brain mapping 2020.

129. Fadaie F, Lee HM, Caldairou B, et al. Atypical functional connectome hierarchy impacts cognition in temporal lobe epilepsy. Epilepsia 2021.

130. Vaessen MJ, Jansen JF, Braakman HM, et al. Functional and structural network impairment in childhood frontal lobe epilepsy. PloS one 2014;9:e90068. 


\section{FIGURES}

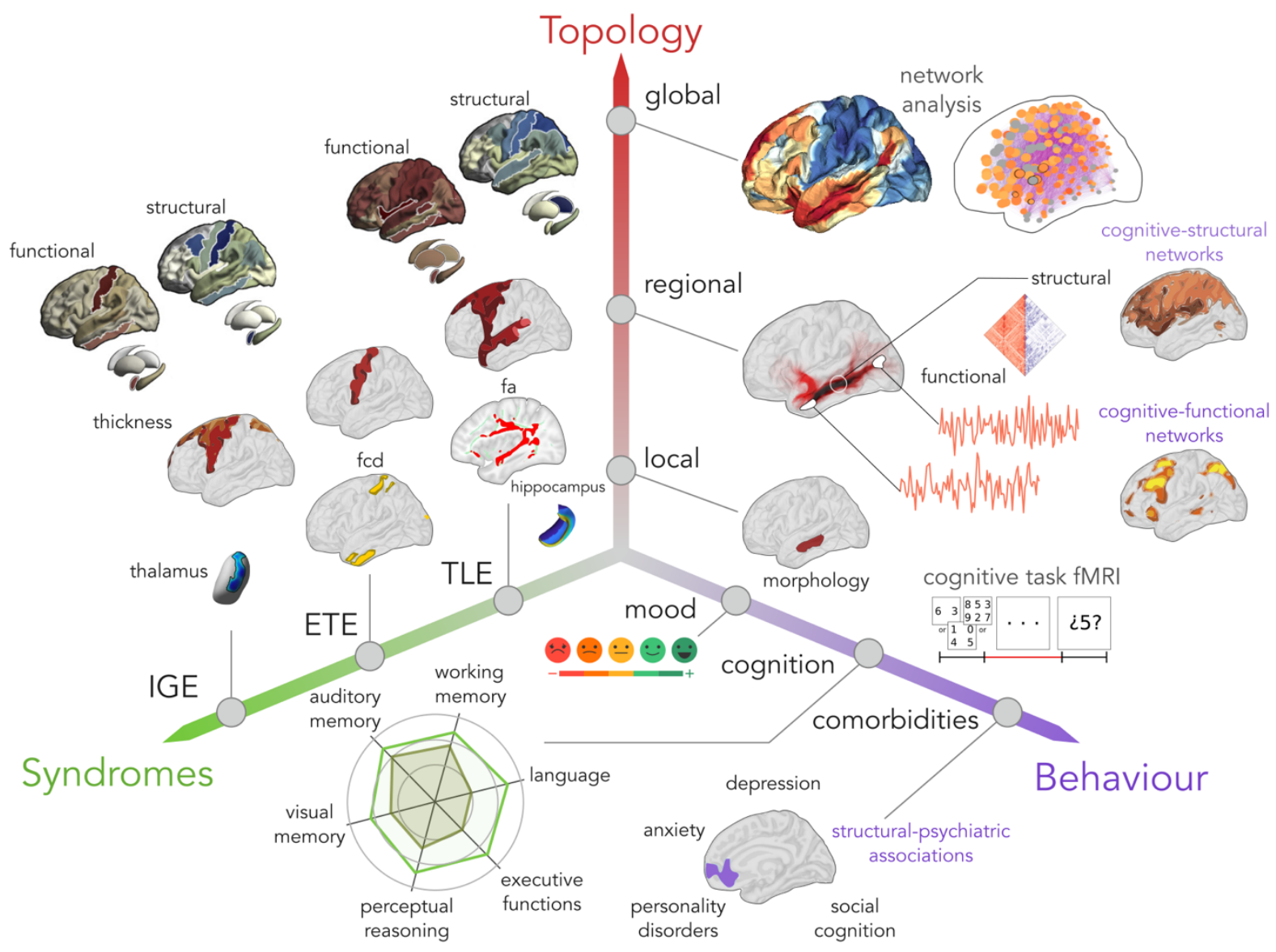

Figure 1. Studying cognitive and affective disfunction across the common epilepsies requires an integrative approach, combining multidimensional behavioral phenotyping, multimodal neuroimaging, and ideally the incorporation of several common epileptic syndromes. In the current review, we will cover the literature that has so far populated this space. 

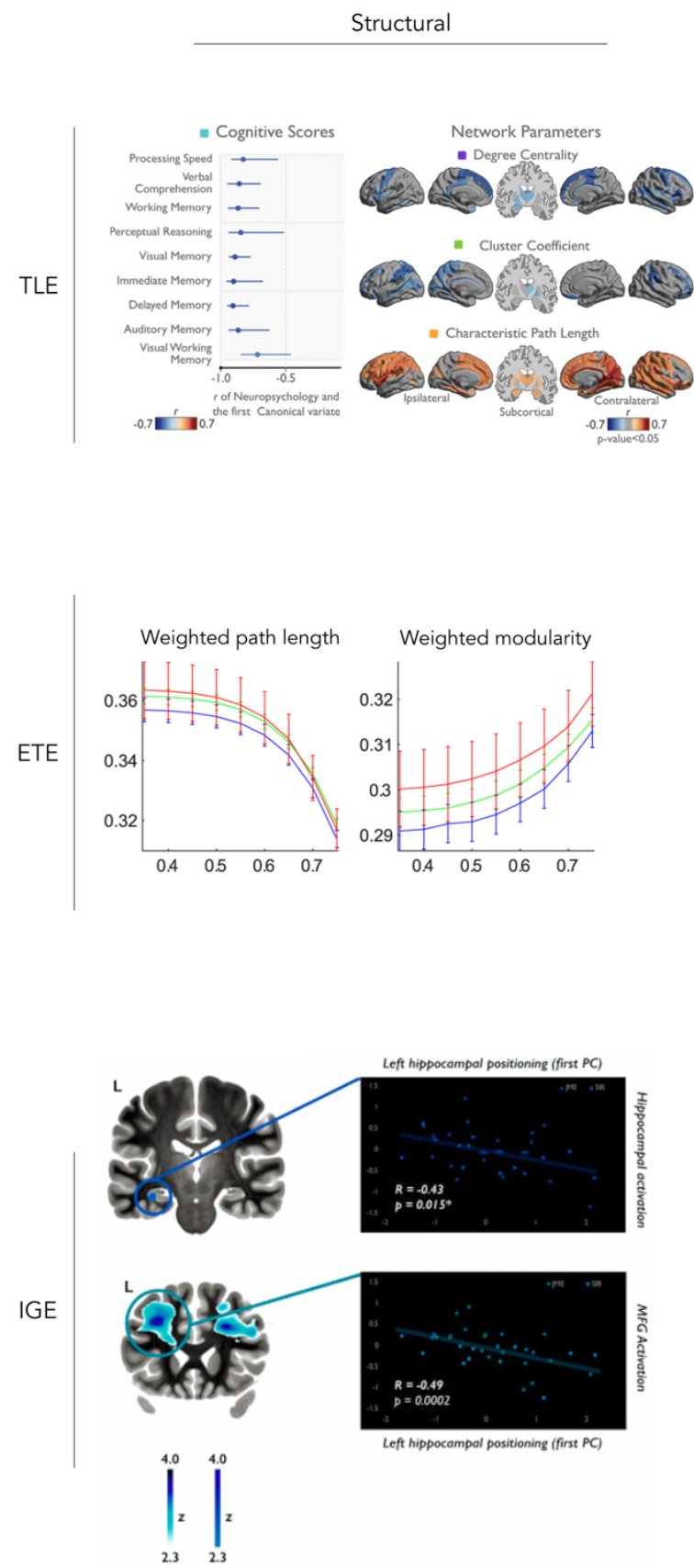

Functional
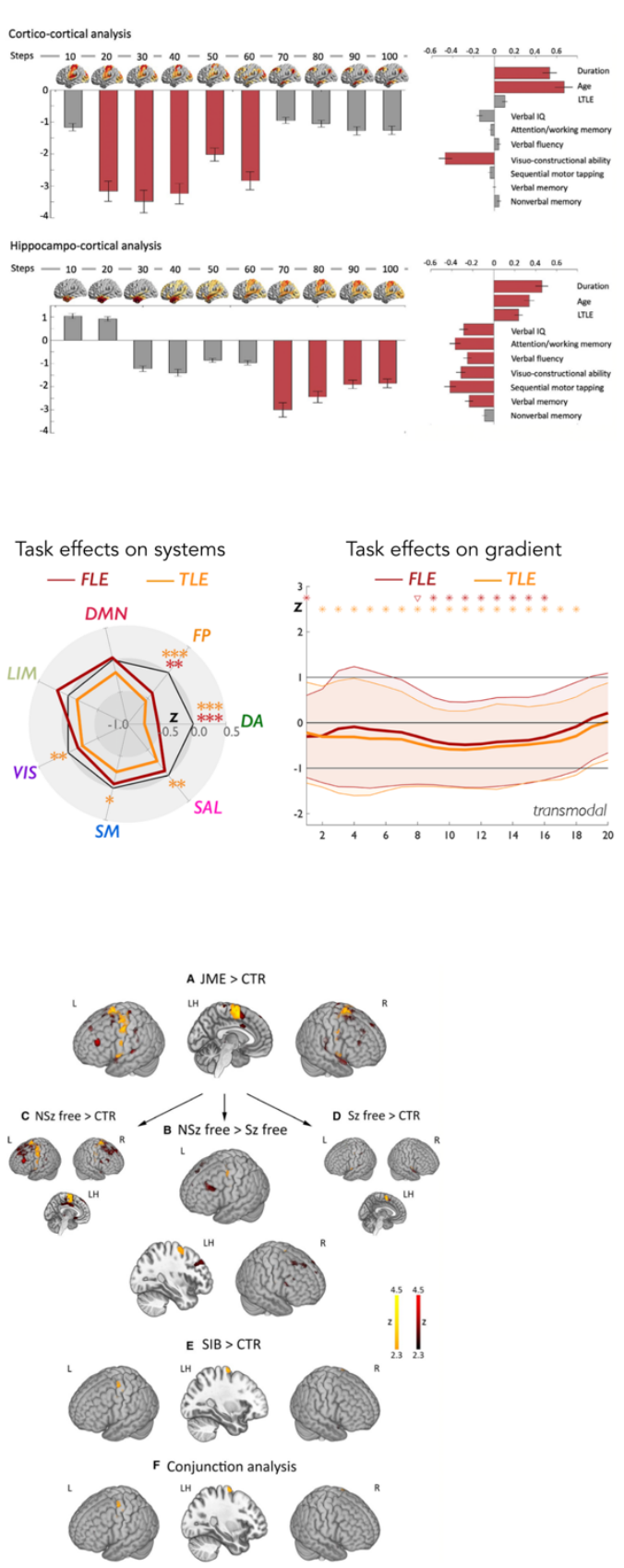

Figure 2. Prior multimodal imaging studies on connectome markers of dysfunction in the common epilepsies. In TLE (top row), structural network measures were associated to cognitive dysfunction across multiple domains (top left) ${ }^{62}$. Using stepwise functional connectivity analysis, alterations in hierarchical functional network organization were shown to reflect multi-domain cognitive impairment (top right) ${ }^{129}$. In ETE (middle row), a study focusing on children with frontal lobe lesions demonstrated that despite the absence of significant differences in structural network parameters in patients, structural modularity increased with stronger cognitive impairment (middle left) ${ }^{130}$. Additionally, when comparing fMRI activation patterns in a verbal working memory task, frontal lobe epilepsy patients showed targeted reductions in the recruitment of specific networks, notably fronto-parietal and dorsal attention systems, while effects in TLE patients were more widespread (middle right) ${ }^{101}$. As for IGE (bottom row), a study in patients with juvenile myoclonic epilepsy and their unaffected siblings studied left hippocampal shape/positional anomalies, and found associations to atypical activation during a verbal memory task (bottom left) 
${ }^{125}$. An endophentype of atypical activation in patients and their siblings could also be seen when assessing both memory and language tasks combined (bottom right) ${ }^{120}$. 